\title{
Signal Acquisition of IRNSS Signals
}

\author{
Dr. S. Muthukumar, \\ HOD: ECE | SVCE, \\ Sriperumbudur, India - 602117
}

\author{
D. Jagadeeshwaran, V. Saveetha, \\ M. K. Subramaniya Raman \\ SVCE, \\ Sriperumbudur, India - 602117
}

\begin{abstract}
1. Abstract:- Indian Regional Navigational Satellite Service (IRNSS) is an independent regional navigation satellite system developed in India. It is designed to provide accurate positioning information service to users in India as well as the region extending up to $1500 \mathrm{~km}$ from its boundary. It will give India its very own indigenous navigation system, which would provide information on location and time in all weather conditions. Our paper discusses about building a software based IRNSS receiver to simulate the navigation behaviour in real time. Navigation using the Indian Regional Navigation Satellite System (IRNSS) requires processing of the lowest raw data received from the corresponding satellites. The acquired Radio Frequency signal is subjected to a software based IRNSS receiver consisting of a Numerically Controlled Oscillator to convert from Radio Frequency Range to Intermediate Frequency Range for further processing. The converted signal is then subjected to various algorithms for achieving Code Acquisition, Locking and Tracking to complete the navigation. All the simulation codes are written in VHDL using VIVADO Software and in accordance with the Zynq-706 FPGA board.
\end{abstract}

The process starts with generating a virtual signal transmitting from IRNSS satellites in the Radio Frequency range. It is then sent to a receiver to acquire and track the signal. This project focuses on

two methods for signal acquisition, namely, Serial Acquisition and Code Phase Parallel Acquisition. [20]

When serial acquisition technique is used, the intermediate frequency signals are convoluted and cross-correlated whereas when parallel acquisition technique is used, both the incoming signal and the locally generated signal is converted to frequency domain, after which a simple multiplication produces the desired effect. Parallel Acquisition also eliminates either of the two feedback loops used in case of serial acquisition, thus further reducing the acquiring time.

After the signal is acquired, a tracking loop is used to fine tune the connection between the satellite and the receiver. This uses feedback loops including Phase Lock Loop, Frequency Lock Loop and Delay Lock Loop.

Research in navigation using IRNSS is in the initial stage. Hence, this paper aims at simulating the real-time behaviour. The obtained results can be used to model the timing constraints and resource allocation for an accurate synthesis of the same and ultimately build an independent navigation system for India.

\section{LITERATURE SURVEY}

A thorough Literature survey was carried out and several technical journals and dissertations were referred.

Navigation is the art of maintaining position and course through traditional practice, geometry, astronomy or specialized tools, which gained its popularity through the Medieval period. In the present scenario, it has become an integral part of the human conscience. Now, there is an absolute need to find and keep track of locations from time to time whenever necessary. This demand for position computation has exponentially increased in the last few decades which led to bolster the focus towards building and developing state-of-the-art devices for the purpose of navigation. [20]

Navigation begins its saga with the advent of locating via magnetic compasses way back in $200 \mathrm{BC}$. Since then, there were a myriad of inventions like the Gyro Compasses and Radars that overtook their predecessors. Finally, the presentday Satellite Navigational Systems took their place to successfully become the most widely used means of navigation. [1] Those of the first commercially available devices were produced almost 2 decades ago, coming up with a technique of serial algorithm for code acquisition in the time domain. Since the resources were limited back then, the only concentration was to utilize the least amount of resources to achieve a functionally accurate system.[4] Although building such a device was successful, the same could not be considered a viable commercial device mainly due to its least time efficiency. That led to the inception of a fast acquisition technique that used frequency domain computations, Global Positioning System (GPS) was the first technology to use such techniques.

However, only in 2013, GPS had an upgrade that led to the launch of additional GPS signals to be available in full constellation. These signals have longer range codes, resulting in increased search space and computational complexity. The code phase and carrier Doppler are estimated based on the different acquisition techniques based on FFT (Fast Fourier Transform). The results show that the acquisition of such an algorithm turns out to have less FFT computation and is an efficient method for different Doppler effects and code phase. [5][3]

When the GPS became highly popular about half a decade ago, many new algorithms were being proposed to further optimize the search process. In 2016, a paper was published that concentrated on CDMA (Code Division Multiple Access) receiver's acquisition and tracking algorithms. Additional effects such as the High dynamic scenario were also taken into consideration according to which, the doppler shift may vary within the range of $\pm 60 \mathrm{kHz}$; Doppler rate may be up to $\pm 66 \mathrm{~Hz} / \mathrm{s}$ and the orbital and attitude control systems may cause a jerk of $10 \mathrm{~g} / \mathrm{s}$. [20] 
With the huge success of universal navigation system, the concentration is next given to reduce the search time and improve the tracking capabilities. A notable model manifesting such requirements is produced in 2018 with an accurate acquisition method of GPS Signal using SDR and Kalman filters. According to which, the received signal from the GPS satellites is digitized through SDR. [5] Then, the subframes and data on navigation are obtained from the phase transition of the navigation data. The hardware collects the digitized data and the user position is discussed in the software. Finally, a Kalman filter of variable bandwidth loop is used to reduce the carrier threshold to mitigate the receiver noise and adapting the loop bandwidth to $\mathrm{CNR}$. [7][3]

The search algorithms for GPS navigation signals were successful only with a rigid constraint of an accurate residual frequency estimation. Hence, 2018 was considered prime time to introduce an algorithm for Residual Frequency Estimation Method for GNSS Receivers.[2][1] Previous estimation methods had several disadvantages, including noise sensitivity and wide search space size. Hence, the article proposed a new method of estimating residual frequency depending on the processing of differentials. This paper is an extension to that final research.[1]

From the above literature survey, we see that several methods have been described for signal acquisition both serially and parallelly. However, for the IRNSS signals, still a lot of exploration needs to be done as it has not been explored thoroughly before. This paper is aimed to simulate in real time, the serial and parallel acquisition as well as tracking of IRNSS signals and further advance into the development of an independent navigational module for India.

\section{MOTIVATION}

During the Kargil war in 1999, one of the primary things that the Indian military required was to get hands on the GPS data of that region as the Pakistani troops took their positions in high mountains. But, due to the denial of access of GPS to India by the United States, GPS could not provide any vital information. The crisis and experience at Kargil war made the country realize the importance of indigenous navigation system and thus, NavIC began to take shape. Also, with other countries like America (GPS), China (BEIDOU), Russia (GLONASS), Japan (Quasi-Zenith), ESA (Galileo) coming up with their own navigation system exhibiting self-reliability in the navigation field created the demand to develop an independent regional navigation system in India.

\section{SIGNAL STRUCTURE}

\section{A. Transmitter}

Signals are transmitted by the IRNSS satellites on two carrier frequencies called as L5 (1176.45 MHz) and S-band $(2472.5 \mathrm{MHz})$ frequencies. The navigation information is transmitted as DSSS which is modulated by a unique PRN sequence belonging to the family of C/A codes of Gold sequence with 1023 bits. Each of these PRN codes is transmitted with the same carrier frequencies through CDMA scheme. [6]

With an intention of detecting and tracking a specific satellite, CDMA scheme should be used to find out the C/A code phase and Doppler frequency shift from the L5 carrier of a specific satellite so that a replica of PRN sequence and carrier wave can be generated at the receiver with the identified code phase and Doppler shift to bring down the desired navigation message to baseband. [7, 8] It is evident from Fig. 1 that the resulting L5 and S-band signals are generated from the same atomic clock with a reference frequency $f_{0}=10.23 \mathrm{MHz}$. This reference atomic clock provides timing to every other signal generator block in the system. The L5 signal is the resultant of the addition of bitwise XOR operation between $\mathrm{C} / \mathrm{A}$ code and navigation and bitwise operation between Precision code $(\mathrm{P})$ and navigation message. After their addition, they are modulated into BPSK onto the L5 carrier having a $90^{\circ}$ phase offset between them.

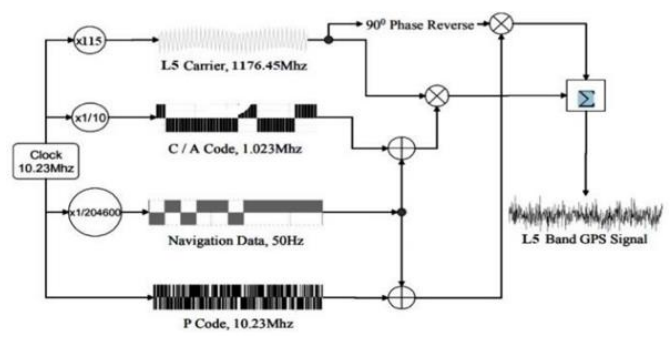

Figure 1

\section{B. Down Sampling}

The initial step of the receiver takes place in the frontend section is to down-sample the Radio Frequency signal to Intermediate Frequency signal as depicted in Fig. 2. It is done because the L5 carrier frequency (1176.45 MHz) is out of range to handle most of the electronic components. Therefore, the RF signal should be down-converted to IF before processing through the receiver. The digital receiver also uses the narrow front-end system bandwidth to improve the performance. $[2,10]$

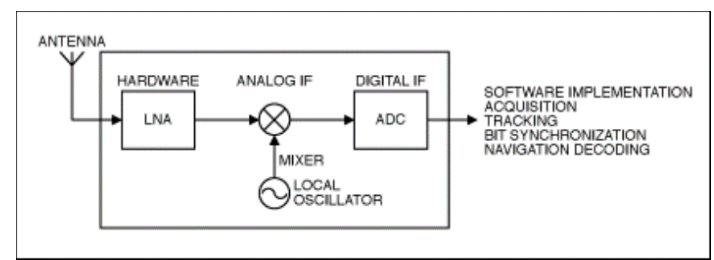

Figure 2

\section{COMPONENTS}

\subsection{PRN CODE GENERATOR}

The spreading sequences used in IRNSS system belonging to a unique family of PRN code are called as Gold codes. Every PRN code that is transmitted by a satellite is a product obtained from the modulo- 2 addition of two $n$ maximum length sequences having a length $\mathrm{N}=2-1$ (in case of IRNSS, $\mathrm{n}=10$, and $\mathrm{N}=1023$ ). The $\mathrm{C} / \mathrm{A}$ code has a chip rate of 1.023 $\mathrm{MHz}$ is a bi-phase modulated signal. Each chip is $977 \mathrm{~ns}$ (1/1.023 MHz) long. 


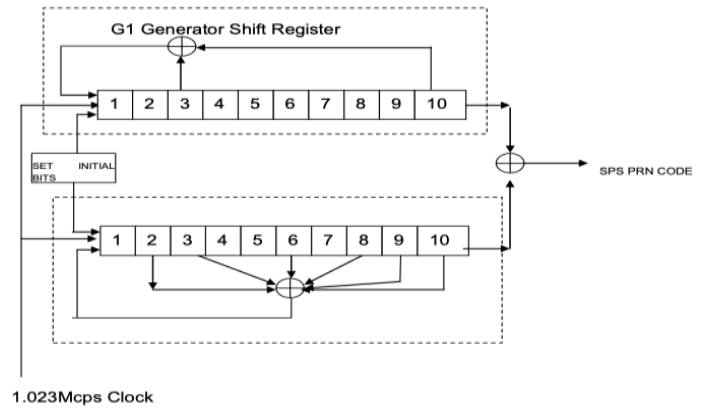

Figure 3

\subsection{NUMERICALLY CONTROLLED OSCILLATOR (NCO)}

$\mathrm{NCO}$ is a digital signal generator used to generate address into a sine lookup table. It is a digital equivalent of Analog VCO. Based on the input phase word, the output frequency changes. The input phase word determines the output frequency of NCO. The carrier NCO is used to generate complex sine and cosine signals whose frequency can be modified. Its primary purpose is to eliminate components from the IF carrier and strip away the Doppler frequency. The frequency control word or the phase word is an input that corresponds to the frequency to be generated. It utilizes the look-up table which is an easier and a faster way to realize carrier NCO. The look-up table utilizes the phase angle as an address to store the sine and cos values. The phase word is an input to the phase accumulator and as the accumulator receives the phase words, it drives a clock to start tabulating.

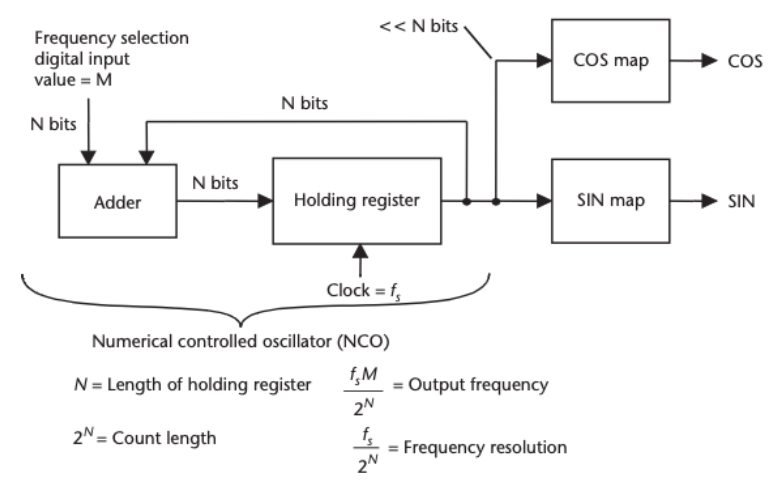

Figure 4

\subsection{ACCUMULATION AND CORRELATION}

The correlator constitutes the most important function of detection and demodulation in the receiver. The digital correlator consists of accumulate and dump in which the signals are multiplied and accumulated. After the multiplication of I and Q components with the PRN code, the signals are sent to the correlator to perform convolution, multiplication and squaring. The maximum index of the Accumulate and Dump block is 1023 . The Accumulate \& Dump performs averaging over one chip rate (adding 1023 values). The average can be positive or negative, but the energy structure after averaging is same as that of I and Q signals. In order to get the energy in absolute value, squaring operation is performed called as enveloping.

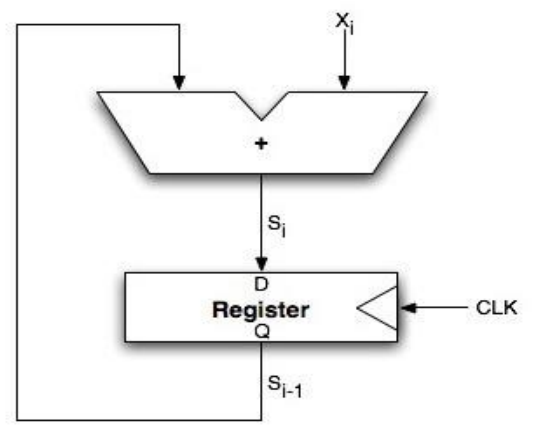

Figure 5

\section{SIGNAL ACQUISITION}

One of the simplest algorithms in the CDMA system used for acquisition is the serial search acquisition. It is one of the classical methods in which the receiver serially searches for the code phase delay within the whole Doppler frequency range. Serial correlation refers to the similarity of the incoming signal with the locally generated signal at the receiver. The primary objective of acquisition is to establish satellite visibility and find the values of carrier Doppler frequency and code phase of the satellite signals. The satellites in the space differ from each other by 32 PRN sequences. It is requisite to know the code offset and Doppler frequency to generate the replica at the receiver. Only when the incoming signal is aligned perfectly with locally generated replica, it is possible to eliminate the incoming code. The line-of-sight velocity of velocity of satellite is the reason for the Doppler Effect which results in lower or higher frequencies. This frequency may vary as much by $\pm 10 \mathrm{KHz}$. Knowing this Doppler frequency is however very crucial to generate a replica at the receiver. On the other hand, if the code phase does not correlate with the locally generated code, then the code phase is incremented by half chip every step which means serial search for 2046 chips is performed. This process performs for a very large number of combinations. Therefore, it is time consuming and exhausting search process which tends to be the main reason for its drawback.

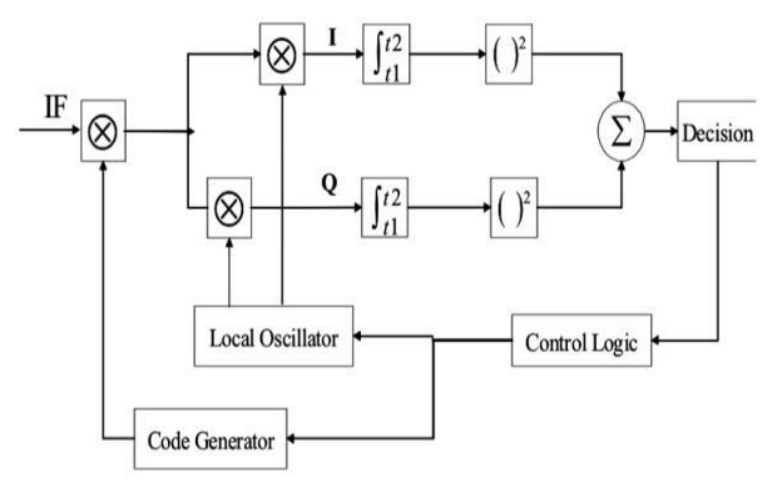

Figure 6 


\section{RESULTS}

\section{A. Serial VS Parallel Acquisition}

The Serial Search and Parallel Search implementations are identical until the component differentiation, after which Serial Search performs linearly and Parallel Search involves converting into Frequency domain before subjecting for further processing. Taking resource usage into constraint, Parallel Acquisition has higher complexity and hence higher usage when compared to serial acquisition due to the involvement of FFT core. Additionally, since the Parallel search tests also several frequency bins simultaneously, it has a higher parallelism. Regarding the Parallel Code Search, it is known that performing a convolution using an FFT is more efficient than with traditional filters when the filter length is more than 64 . However, when considering the complexity of implementation, Serial Acquisition surpasses parallel due to ease in its architecture as illustrated in Table 1.

\begin{tabular}{|c|c|c|}
\hline Algorithm & Iterations & Complexity \\
\hline Serial acquisition & 41943 & Low \\
\hline $\begin{array}{c}\text { Parallel Frequency } \\
\text { Space Search }\end{array}$ & 1023 & Medium \\
\hline $\begin{array}{c}\text { Parallel Code } \\
\text { phase Space } \\
\text { Search }\end{array}$ & 41 & High \\
\hline
\end{tabular}

Table 1

\subsection{NUMERICALLY CONTROLLED OSCILLATOR}

The Fig.7 depicts the generation of sine and cosine waveforms using a carrier $\mathrm{NCO}$ with the help of a look-up table. The step size is represented by the phase error used to generate a clock frequency of $1.26 \mathrm{MHz}$. The upper 3 bits of the accumulator which 30 bits is used as the address to find the corresponding amplitude of waveforms. The sine and cos outputs are passed on to other blocks as I and Q components represented integers.

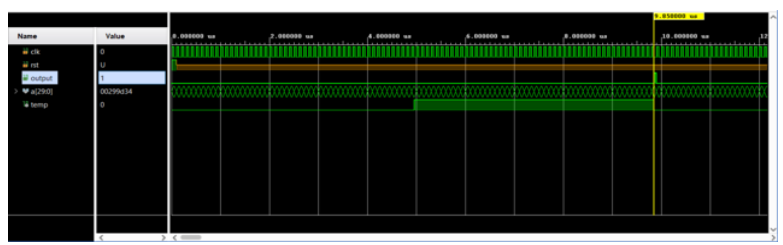

Figure 8

\subsection{SERIAL ACQUISITION}

The data obtained from the RF front end is down converted to IF signal for further processing in the receiver. The sampling clock for the entire receiver processing is $10 \mathrm{MHz}$. The Fig. 13 illustrates the serial acquisition process. Once the signal is down sampled, the IF Signal is multiplied with the C/A code as well as outputs of local oscillator namely loc_sin and loc_cos. These signals are then transmitted into the correlator and finally to a decision block to check if the correlation value exceeded the pre-defined threshold. This implicitly means if the satellite is acquired or not. If the satellite is not acquired, then a delay of half chip is introduced in the C/A code generated by clk_2M as portrayed in Fig.13. This process is performed for 1023 chips until the satellite is acquired.

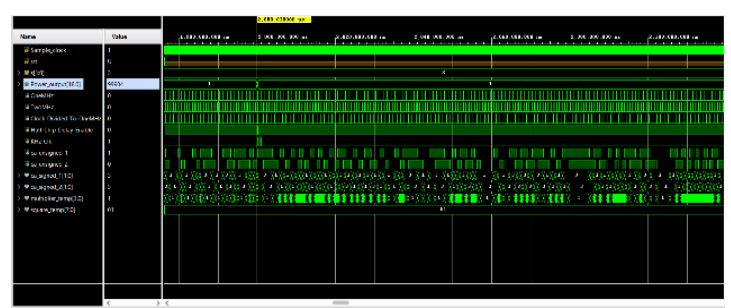

Figure 9

\section{CONCLUSION}

The research began with an overview of the IRNSS signal structure with extra attention to the theories of signal acquisition and signal tracking. The traditional and advanced acquisition methods were discussed. The acquisition is the first phase for processing of the IRNSS signal in the receiver. The goal of acquisition is to measure the code offset and Doppler frequency. The reality is that the receiver often does not know a priori which visible satellites are. A complete description of the process of acquisition and two popular methods that we could use is provided. The two important parameters produced by the acquisition are the carrier frequency and the initial C/A code phase. The serial search algorithm sequentially quests for a satellite at every possible code phase and Doppler frequency in the time domain. Since there is only need for additional and multiplication operations, time domain execution is straightforward. Since the serial search algorithm must do a huge amount of glide correlation in a real-time IRNSS receiver, the biggest flaw of the serial search acquisition also appears to be this exhausting correlation. We could deduce that serial acquisition is a quite simple algorithm, but presents many hurdles in the acquisition process, more specifically due to its time consumption.

\section{REFERENCES}

[1] B.R. Iswariya, H. Nirosh Kumar, "FFT based Acquisition techniques of GPS L2C signals", ICTACT Journal on Communication Technology, vol. 04, no. 04, pp. 849-853 Dec. 2013.

[2] Sandip Sudani, Dhaval Upadhyay, Pravin Patidar, "Implementation of Acquisition \& Tracking Algorithms of Spread Spectrum Signals in high dynamic scenario", International Journal of Current Advanced Research, vol. 5, no. 3, pp 643-648, March 2016.

[3] A Pirovano, A Redaelli, F Pellizzer, F Ottogalli, M Tosi, D Ielmini, A L Lacaita, R Bez, "Acquisition and Tracking of NavIC L5 band signals", International Journal of Applied Engineering Research, vol. 12, no. 19, pp 8115-8119, Dec. 2017

[4] N.R. Raajan, G.Madhu Priya, K.S.Lavanya, S.Raghavi, S.Greeta, V.S. Ramya Lakshmi, K.Hariharan, "Analyzing the Acquisition And Tracking in GPS transceiver using SDR and Kalman filter", International Journal of Pure and Applied Mathematics, vol. 19, no. 12, pp 16391-16402, Jan. 2018.

[5] Hao Cui, Zhigang Li, Zheng Dou, "Fast Acquisition Method of GPS Signal Based on FFT Cyclic Correlation", International Journal. Communications, Network and System Sciences, vol. 10, no. 20, pp 246-254, Aug. 14, 2017. 
[6] Dinesh MANANDHAR, Yongcheol SUH, Ryosuke SHIBASAKI, "GPS Signal Acquisition and Tracking-An Approach towards Development of Software-based GPS Receiver", Technical report of IEICE

[7] Pooja V, Thakar, Hiren Mewada, "Receiver Acquisition Algorithms and their comparisons for BOC Modulated Satellite Navigation System", International Conference on Communication Systems and Network Technologies.

[8] Tsui, J.B-Y, Fundamentals of Global Positioning System Receivers, John Wiley and Sons, 2000, ISBN 0-471-38154-3

[9] Y. Luo, L. Zhang and H. Ruan, "An Acquisition Algorithm Based on FRFT for Weak GNSS Signals in A Dynamic Environment," IEEE Communication Letters, vol. 20, no. 03, pp. 1-4, 2018.

[10] Moreau, M., Axelrad, P., Garrison, J.L., Kelbel, D., and Long, A., "GPS Receiver Architecture and Expected Performance for Autonomous GPS Navigation in Highly Eccentric Orbits," Proceedings of the ION 55th Annual Meeting, pp. 653-665, June 28-30.

[11] Y.-H. Chen, S. Lo, D. Akos, P. Enge, "Direct Comparison of L1 BOC $(1,1)$ vs L1 C/A multipath performance Using On Air Galileo and QZSS transmissions" Proceedings of the Institute of Navigation/Institute of Electronics and Electrical Engineers Position Location and Navigation Symposium (PLANS), May 2014.

[12] S. Wang, L. Gao, J. Bai and F. Ye, "Assisted GPS Signal Acquisition Algorithm Based on the Joint Code-frequency Search" in Progress in Electromagnetics Research Symposium, Singapore, vol. 57, no. 06, pp 19-22, 2017.

[13] J. Leclere, C. Botteron and P.-A. Farine, "Comparison Framework of FPGA-Based GNSS Signals Acquisition Architectures," IEEE Transactions on Aerospace and Electronic Systems, vol. 49, no. 3, pp. 1497-1518, 2013.

[14] Hao Cui, Zhigang Li, Zheng Dou, "Fast Acquisition Method of GPS Signal Based on FFT Cyclic Correlation", International Journal. Communications, Network and System Sciences, vol. 10, no. 20, pp 246-254, Aug. 14, 2017.

[15] Zhang Zhong, XueBingbing. "Pseudo-code Tracking Technology in Spread Spectrum Communication", Fourth International Conference on Computational Intelligence and Communication Networks, vol. 13, no. 81, pp 331-333, Jan. 2012.

[16] QIU LIE and Li LIE, "GPS Signal Acquisition based on FFT", Second international Conference on Information Technology and Computer Science, vol. 30, no. 06, pp 1005-1008, 2015.

[17] T. H. Ta, M. Pini, L. Presti, "Combined GPS L1C/A and L2C signal acquisition architectures leveraging differential combination," IEEE Transactions on Aerospace and Electronic Systems, vol. 50, no. 04, pp 3212-3229, 2014.

[18] C. Wu, L. Xu, H. Zhang and H. Zhang, "An Improved Acquisition Method for GNSS in High Dynamic Environments: Differential Acquisition Based on Compressed Sensing Theory," Navigation: Journal of The Institute of Navigation, vol. 64, no. 1, pp. 23-34, 2017.

[19] Jianfeng Wu. "The Study on GPS Signal Acquisition Algorithm in Time Domain", 2008 4th International Conference on Wireless Communications Networking and Mobile Computing, vol. 23, no. 43, pp 1-3, Feb. 2012

[20] 20. 1. Kaplan, E. "Understanding GPS: Principles and Applications", Norwood, MA, Artech House, 1990 